\title{
Lidil
}

Revue de linguistique et de didactique des langues

$61 \mid 2020$

Le mépris en discours

De l'expression du mépris au marquage d'une solidarité : l'emploi ontotypique de cassos, beauf, bolos, crevard, kéké et kikoo dans les discours spontanés entre jeunes

From the Expression of Contempt to the Marking of Solidarity: The Ontotypic Use of cassos, beauf, bolos, crevard, kéké and kikoo in Students Spontaneous

Discourse

\section{Polina Ukhova}

\section{CpenEdition}

Journals

Édition électronique

URL : http://journals.openedition.org/lidil/7274

DOI : $10.4000 /$ lidil. 7274

ISSN : 1960-6052

Éditeur

UGA Éditions/Université Grenoble Alpes

Édition imprimée

ISBN : 978-2-37747-195-9

ISSN : $1146-6480$

Référence électronique

Polina Ukhova, « De l'expression du mépris au marquage d'une solidarité : l'emploi ontotypique de cassos, beauf, bolos, crevard, kéké et kikoo dans les discours spontanés entre jeunes », Lidil [En ligne], 61 | 2020, mis en ligne le 02 mai 2020, consulté le 02 mai 2020. URL : http://journals.openedition.org/ lidil/7274; DOI : https://doi.org/10.4000/lidil.7274

Ce document a été généré automatiquement le 2 mai 2020.

(C) Lidil 


\section{De l'expression du mépris au} marquage d'une solidarité : l'emploi ontotypique de cassos, beauf, bolos, crevard, kéké et kikoo dans les discours spontanés entre jeunes

From the Expression of Contempt to the Marking of Solidarity: The Ontotypic Use of cassos, beauf, bolos, crevard, kéké and kikoo in Students Spontaneous Discourse

Polina Ukhova

\section{Introduction}

1 Les philosophes et les anthropologues ont depuis longtemps contribué au développement des questions liées à l'impact des émotions sur nos décisions comportementales et nos relations sociales. Mais ce n'est que depuis quelques années que les linguistes francophones s'intéressent à l'expression verbale des émotions. Nous pensons, plus particulièrement, à de nombreuses études sur la violence verbale (Moïse, 2004), sur le discours de dérision et sur les insultes (Lagorgette \& Larrivée, 2004 ; Lagorgette, 2007), sur le discours polémique (Amossy, 2014), sur la médisance (Mougin, 2006), sur les injures (Rosier, 2006), sur les modèles d'analyse des émotions en discours (Micheli, 2014). L'acte de mépris, dont les manifestations linguistiques sont souvent difficiles à cerner, s'avère moins étudié.

2 L'objectif de cette étude est d'analyser le fonctionnement de quelques lexèmes non standard (à savoir cassos, beauf, bolos, crevard, kéké et kikoo) dans les discours entre étudiants de 18 à 23 ans. Nous chercherons à saisir comment l'actualisation de ce vocabulaire à substrat idéologique et socioculturel dans des contextes à portée 
condescendante contribue à traduire le contenu méprisant et identifierons des conditions nécessaires pour la réalisation d'un acte de mépris. Nous nous intéresserons également à des contextes de solidarité à visée affective où l'apport sémantique du lexique en question sera relativisé et essaierons de décrire les procédés de ce retournement en contre-discours affectif à travers le prisme de l'intersubjectivité. Notre étude se fonde sur les données de deux corpus de parler d'étudiants qui seront appuyées par les résultats d'un questionnaire sociolinguistique. Nous tenterons de dégager les particularités pragmatiques de l'acte de mépris et les effets qu'il provoque chez soi et chez autrui.

\section{Présentation des corpus}

3 La population choisie pour cette étude est celle des étudiants. Elle regroupe des jeunes de 18 à 23 ans qui ont été scolarisés et qui poursuivent au moment de l'expérimentation leurs études universitaires. Nous avons constitué deux corpus : un corpus oral, que l'on peut qualifier d'«écologique» puisqu'il s'agit de 18 heures d'enregistrement de conversations spontanées entre étudiants de l'université de Poitiers ${ }^{1}$ et de 30 heures d'enregistrements radiophoniques (nous avons choisi l'émission «Guillaume Radio 2.0 " sur NRJ, chaine radiophonique française, car l'animateur indique systématiquement l'âge de chaque auditeur qui appelle lors des émissions, ce qui nous a permis de rester fidèle à la tranche d'âge choisie), ainsi qu'un corpus écrit, constitué d'occurrences relevées sur les réseaux sociaux (Facebook, WhatsApp), représentatif de l'écrit oralisé2.

\section{Cadre théorique}

\subsection{Le mépris d'un point de vue axiologique}

4 Il semble logique de commencer notre propos par la définition de la notion-clé qu'est celle de mépris. Comme l'indique le Dictionnaire Larousse,

le mépris est un sentiment par lequel on juge quelqu'un ou sa conduite moralement condamnables, indignes d'estime, d'attention, une attitude, sentiment par lesquels on s'élève au-dessus de ce que généralement redoutent les autres hommes, ou de ce qui les attire.

Mépriser signifie considérer quelqu'un, sa conduite comme indignes d'estime, de considération, les condamner moralement ou ne faire aucun cas de quelque chose, le négliger ${ }^{3}$.

5 L'acte de mépris suppose donc que le locuteur exprime un jugement sur autrui lui permettant de s'élever au-dessus; autrement dit, il s'agit à la fois de la dévaluation de l'autre et de la valorisation de soi lors de la production de l'énoncé. Nous nous retrouvons donc dans le domaine subjectif des valeurs ${ }^{4}$. De ce fait, plusieurs remarques s'imposent.

D'abord, en parlant des jugements de valeur, la première notion à convoquer est celle de norme. Pour juger que quelque chose est bien ou mal, il faut se représenter une norme qui servirait de référence. Ensuite, le jugement ne peut pas être posé si l'objet évalué n'appartient à aucune catégorie d'objets, c'est-à-dire que la question qui porte sur l'évaluation ne peut pas se poser simplement comme ceci: «Est-ce bon ou 
mauvais ? ", mais qu'elle doit prendre en compte la taxonomie axiologique de l'objet, c'est-à-dire «X est-il bon ou mauvais pour quelque chose ?»; par exemple : « ce chien est-il un bon cadeau pour un chasseur? » (pour quelqu'un qui n'est pas chasseur, pour un ami qui loue un petit studio etc.).

7 Selon Perry (1926), tout ensemble d'objets ne représente pas pour autant une classe comparatiste. Il n'existe pas de critères pour les classes qui sont constituées de manière arbitraire ni pour celles qui ne contiennent qu'un seul élément. Par exemple, l'adjectif bon ne s'associe ni avec les noms propres ni avec les termes géographiques. Il serait impossible de décider si le pôle Nord est bon ou mauvais, pourtant en tant qu'élément de la classe " habitats de l'être humain ", il peut être jugé comme pire ou meilleur que le bassin méditerranéen. Nous nous appuyons ici sur les réflexions de Perry (1926) et de Hare (1999), qui débouchent sur la notion de motif qui contribue à l'interprétation des jugements de valeur. Nous y reviendrons.

8 Notons par ailleurs que pour tout acte réussi, il est indispensable que les interactants aient le même univers moral et idéologique et, donc, la même conception de la norme. Quand la norme est admise par tout le monde, il est inutile de l'expliciter. En revanche, si le locuteur se base sur un standard nouvellement adopté ou personnel, il est souhaitable de l'indiquer afin de faciliter l'interprétation de l'énoncé.

9 Ainsi, pour interpréter un énoncé comme acte de mépris, la scénographie énonciative (construite par le texte lui-même et le contexte de sa production ${ }^{5}$ ) doit s'y prêter. Si le jugement que le locuteur porte sur son destinataire afin d'exprimer son sentiment de mépris n'est pas compris ou n'est pas perçu comme tel, l'effet souhaité ne sera pas atteint. Citons un bref exemple d'un acte de mépris échoué tiré de notre corpus écrit :

(1)

L1 : si je te parle plus $\mathrm{c}$ qu il y a une raison

$\mathrm{L} 2$ : Vas y balance quoi que jen ai rien à foutre de se qu tu penses de moi

$\mathrm{L} 1: \mathrm{T}$ qu'une connasse en fait les gens comme toi me dégoute pffff

L2: Oh que ouiiiii (EF123) ${ }^{6}$

Dans (1), le jugement évaluatif «t qu'une connasse » est perçu par le destinataire comme mélioratif et non pas comme méprisant. «Le dégout » ressenti par le locuteur serait voulu et peut-être même intentionnellement provoqué par son interlocuteur qui voit son objectif atteint et incorpore le jugement de valeur comme un compliment («Oh que ouiiiii »). En revanche, l'intention communicative du locuteur n'a pas abouti, car l'effet perlocutoire du mépris n'a pas été réalisé.

11 Soulignons que tout jugement peut également provoquer un débat qui ne se résume pas à un désaccord entre deux motifs, mais à une divergence d'intérêts. Si l'un des interlocuteurs affirme « Il vaut mieux aller au cinéma » et que l'autre le contredit en lui proposant d'aller à un concert, les deux peuvent avoir le même motif (celui d'un passetemps agréable).

12 En résumé, insistons sur le fait que tous les paramètres de la situation énonciative sont impérativement à prendre en compte pour traiter des actes de mépris, à savoir : sujet (énonciateur), objet (cible évaluée), motifs (un point de vue corrélé à l'échelle des normes morales, comportementales, socioculturelles). Notons également que celui qui amorce la situation de communication et qui produit un ethos dominant par son discours est aussi important que l'interlocuteur qui assimile les informations linguistiques et extralinguistiques reçues avant de réagir. 
13 Suivant Maingueneau (2009), le terme d'ethos, qui est très important pour tout acte réussi, sera défini comme

ce que l'orateur prétend être, il le donne à entendre et à voir : il ne dit pas qu'il est simple et honnête, il le montre à travers sa manière de s'exprimer. L'ethos est ainsi attaché à l'exercice de la parole, au rôle qui correspond à son discours, et non à l'individu réel appréhendé indépendamment de sa prestation oratoire (p. 138).

Il peut être produit implicitement, mais aussi explicitement ( je suis quelqu'un de posé »). C'est la différence qu'Amossy (2010) explique en deux termes, à savoir le dit et le dire. Pour elle, le dit est ce que l'énonciateur énonce explicitement sur lui-même, alors que le dire émerge de son discours même s'il ne se réfère pas à lui-même. Nous partageons aussi l'avis de Maingueneau (2009) qui ne limite pas l'analyse de l'ethos aux textes oraux, en postulant que même les textes écrits possèdent un ton permettant au coénonciateur de définir plus ou moins les caractéristiques de son énonciateur. Les données de notre corpus écrit seront donc également analysées dans le cadre de cet article.

Suivant Alsafar (2014), notons également que le même message peut être perçu différemment selon la situation énonciative englobante (qui définit le statut des partenaires et un cadre spatio-temporel), générique (attachée à un genre ou un sousgenre de discours) et scénographique (construite par le texte ou en fonction des paramètres de la situation énonciative). Dans notre étude, la situation énonciative englobante se présente sous forme de discours non planifiés à l'oral et à l'écrit entre locuteurs de même âge, la situation générique, sous forme de conversations du quotidien qui tournent autour des loisirs et des préoccupations des jeunes et la situation scénographique se présente sous trois modalités: discussions en face à face, échanges numériques, conversations radiophoniques.

La situation énonciative avec ses modalités et ses paramètres doit nécessairement être prise en compte pour assurer le décodage correct des énoncés. Par exemple, si l'on se réfère à l'étude effectuée par Conein (2017, p. 147) à partir des données du Multicultural Paris French Corpus (désormais MPF), nous voyons que dans les milieux multiculturels, les termes désignant la nationalité servent de moyen d'«énoncer son identité " par rejet de l'identité d'autrui et fonctionnent souvent comme " des quasi-noms d'ethnie ». Il s'agit de situations d'énonciation où en disant, par exemple, "je suis algérien " le locuteur vise à faire entendre " je ne suis pas français ». En revanche, si l'on analyse les données de nos corpus, nous voyons que dans le milieu étudiant, les termes comme babtou (qui fonctionne comme synonyme de fragile, sensible sans aucun rapport avec la couleur de la peau ${ }^{7}$ ) perdent leur dominante et sont utilisés à des fins humoristiques ou ironiques. Dans cette optique, nous pourrions supposer que dans certains contextes propices à la production d'identité, en traitant quelqu'un de kéké ou de cassos, le locuteur, en posant un ethos méprisant, prétendrait ne pas l'être, et dans d'autres, à portée ludico-conniventielle, aurait l'intention de provoquer le rire. Cette hypothèse sert de point de départ pour notre étude. Nous y reviendrons.

\subsection{Typologie de référence}

17 Notre analyse porte sur le lexique qui est potentiellement envisageable comme méprisant par son sémantisme. Pour cette raison, plusieurs termes (cassos, beauf, bolos, crevard, kéké et kikoo) ont été retenus pour l'étude. Or, nous verrons qu'ils ne sont pas 
toujours employés dans des contextes dévalorisants afin d'exprimer la force illocutoire du mépris. Pour que ce lexique soit considéré comme méprisant et qu'il puisse provoquer un effet pragmatique, il faut que le destinataire l'identifie comme tel en s'appuyant sur un ensemble diffus de représentations sociales évaluées positivement ou négativement, de stéréotypes que l'énonciation contribue à conforter ou à transformer (Maingueneau, 2009).

De ce fait, notre analyse se fondera sur deux approches, à savoir pragmatique et intersubjective. Elle prend appui sur la typologie des représentations idéologiques et identitaires se manifestant dans le discours, car nous nous intéressons au lexique à fort substrat idéologique. Cette classification distingue trois catégories qui conceptualisent des manières de se dire soi, mais aussi et surtout d'être interpellé comme individu dans son identité (nationale, sociale, sexuelle ou essentielle) : ethnotypes (Bres, 1993) l'Arabe, le Belge, le Rital -, sociotypes (Bres, 1993) - le bourge, le cassos, le beauf - et ontotypes (Ernotte \& Rosier, 2004) - le kéké, le snob, le crevard.

L'idéologie façonne les représentations que nous avons du monde environnant, et en particulier, de la vie sociale. Suivant Seca (2012), notons qu'

une idéologie est un système prédéfini d'idées, appelées aussi catégories, à partir desquelles la réalité est analysée. Une idéologie est souvent la dimension culturelle d'une catégorie sociale, [...] elle est typiquement imposée [...] de façon imperceptible dans la vie courante (p. 1).

20 Ainsi, pour reprendre notre typologie de référence, les images façonnées de l'appartenance de classe et réalisées dans des discours stéréotypés sont appelées sociotypes. Ces déterminations peuvent affecter les échanges entre interactants de différents groupes sociaux qui engendrent les représentations du même et de l'autre. En ce qui concerne l'ethnotype, c'est l'identité ethnique qui est en cause. Cette catégorie se caractérise par le renforcement de la règle d'axiologisation : la valorisation de soi et la dévalorisation de l'autre lors de la production de l'identité. Les profils ethnosociotypiques se manifestent plus souvent comme le fondement idéologique qui provoque et explique à la fois certaines réactions discursives et comportementales dans des interactions. La troisième catégorie, celle des ontotypes, est proposée par Ernotte et Rosier (2004). Elle regroupe des stéréotypes discursifs qui visent des caractéristiques supposées ontologiques de l'individu (fainéant, relou, etc.). Selon les auteurs, l'ontotype n'est pas sémantiquement fortement marqué par des idéologies si on le compare aux ethnotypes et aux sociotypes. En effet, en traitant quelqu'un de relou, nous ne nous appuyons pas nécessairement sur une norme imposée ou sur des critères communément prédéfinis et admis, contrairement à cassos qui renvoie au critère socioéconomique concret représentant un fondement de ce jugement. Le mécanisme discursif réduit le fonctionnement des jugements ontotypiques à leur contexte situationnel concret (pas toujours direct, mais qui fait référence à une actualité concrète, passée), ce qui les distingue des jugements socio- et ethnotypiques, qui ne dépendent aucunement des causes externes pour être émises (Ernotte \& Rosier, 2004).

21 Reprenons les mêmes items pour illustrer ce constat. Relou en tant qu'ontotype ne peut fonctionner ni être compris s'il est employé hors contexte : il est impossible de déduire, par exemple, s'il s'agit d'une personne ou d'une situation embarrassante; les motifs de ce jugement ne sont pas non plus distinguables. Pour appuyer notre propos, soulignons ici que l'ontotype, selon les auteurs, est rare dans les représentations communes. En effet, si cassos, même coupé de la situation d'énonciation concrète, renvoie toujours, 
en tant que sociotype, à une catégorie sociale de personnes qui se retrouvent dans une même situation bien identifiable (dans ce cas, il s'agit d'une difficulté socioéconomique), relou se comporte différemment : les ontotypes consistent davantage « en des prédications évaluatives qui reposent sur un jugement de valeur alors que les sociotypes visent essentiellement une identification » (Ernotte \& Rosier, 2004, p. 40).

comme le souligne Juignet (2016), la démarche ontologique «n'aboutit pas à un savoir apodictique. Une ontologie est toujours hypothétique et ne peut prétendre à la vérité, mais seulement à la vraisemblance ". Toute conception du réel est sujette à révisions avec l'avancée des observations. Les ontotypes sont donc fortement ancrés dans la situation énonciative et toujours axiologiquement marqués, tandis que les sociotypes peuvent même fonctionner comme des appellatifs neutres (Ernotte \& Rosier, 2004).

Cette typologie nous semble applicable dans le cadre de l'analyse des évaluatifs péjoratifs. Il s'agirait d'une sorte de sociotypification qui interrogerait les normes sociales et culturelles. Nous observerons par la suite comment les sociotypes et les ontotypes fonctionnent dans différents contextes afin de distinguer ceux qui relèvent du mépris. Les ethnotypes ne seront pas analysés dans le cadre de cet article.

\subsection{Présentation du questionnaire}

Afin de délimiter le champ distributionnel des termes cassos, beauf, bolos, crevard, kéké et kikoo et d'identifier des stratégies discursives de mépris utilisées dans différents contextes d'emploi, nous avons mis en place un questionnaire. 50 étudiants de l'université de Poitiers âgés de 18 à 23 ans y ont participé. Nous avons proposé aux enquêtés six lexèmes accompagnés de définitions tirées de dictionnaires de référence ${ }^{8}$. Plusieurs tâches étaient demandées aux participants : a) cocher la/les définition(s) qui leur parait (paraissent) la/les plus exacte(s) (ou proche(s)) de l'emploi actuel; b) nuancer cet emploi en précisant des connotations potentiellement envisageables des lexèmes en question; c) fournir d'éventuels commentaires, précisions et exemples d'emploi.

Les items en question ont été sélectionnés à partir des usages identifiés dans les corpus oraux et écrits. Leur emploi actuel reste flou, contrairement à ce que nombreux ouvrages lexicographiques laissent entendre. Il nous semble que leur analyse d'un point de vue pragmatique pourrait être fructueux, et éclairer notamment les mécanismes discursifs de mépris.

Figure 1. - Consigne du questionnaire.

\begin{tabular}{|l|l|l|l|}
\hline Item & Définitions & Connotation & Exemples et commentaires \\
\hline
\end{tabular}




\begin{tabular}{|c|c|c|c|c|c|}
\hline $\begin{array}{l}\text { Employez- } \\
\text { vous ce mot? }\end{array}$ & $\begin{array}{l}\text { (cochez celle(s) } \\
\text { qui correspond(ent) à } \\
\text { leur emploi actuel) }\end{array}$ & $\begin{array}{l}6 . \\
7 .\end{array}$ & $\begin{array}{l}\text { péjorative } \\
\text { ironique } \\
\text { vulgaire } \\
\text { humoristique } \\
\text { positive/ } \\
\text { appréciative } \\
\text { vulgaire } \\
\text { neutre } \\
\text { (possible de } \\
\text { mettre plusieurs } \\
\text { réponses) }\end{array}$ & 2) & $\begin{array}{l}\text { nuancez/ redéfinissez les } \\
\text { termes si les définitions } \\
\text { données vous paraissent } \\
\text { inexactes ou fausses; } \\
\text { proposez 1-2 phrases pour } \\
\text { montrer le contexte d'emploi } \\
\text { du terme en question que } \\
\text { vous pourriez vous imaginer } \\
\text { dire. }\end{array}$ \\
\hline
\end{tabular}

\section{Résultats de l'enquête}

\subsection{Définitions des termes étudiés}

Nous avons résumé les définitions et les remarques obtenues dans le tableau 1 cidessous :

Tableau 1. - Résultats du questionnaire : définitions et commentaires.

\begin{tabular}{|c|c|c|}
\hline Item & Définitions proposées & CommentAires fournis ${ }^{9}$ \\
\hline CAssos & $\begin{array}{l}\text { Définition } 1(25,6 \%) \text { : } \\
\text { Cas social, personne en grande difficulté } \\
\text { financière ou sociale. } \\
\text { Définition } 2(45 \%) \text { : } \\
\text { Cas désespéré, personne peu intelligente ou } \\
\text { parfois, personne complètement décalée. } \\
\text { Définition } 3(9,4 \%) \text { : } \\
\text { Quelqu'un qui a un style douteux, il s'agit des } \\
\text { gens plus ou moins attardés mentaux. } \\
\text { Définition } 4(20 \%) \text { : } \\
\text { Personne marginale et perturbée. }\end{array}$ & $\begin{array}{l}\text { (Femme, ScPO, } 19 \text { ans) } \\
\text { Au sens premier - personne en grande } \\
\text { difficulté financière ou sociale, cette expression } \\
\text { est plutôt neutre. Le mot a dérivé et est devenu } \\
\text { insultant. } \\
\text { (Femme, M1 linguistique, } 21 \text { ans) }: \\
\text { Insulte pour désigner quelqu'un peu intelligent } \\
\text { dont le comportement est hors norme. } \\
\text { (Homme, LEA, } 20 \text { ans) }: \\
\text { Peut être employé juste comme insulte. }\end{array}$ \\
\hline
\end{tabular}




\begin{tabular}{|c|c|c|}
\hline Beauf & $\begin{array}{l}\text { Définition } 1(9,6 \%) \text { : } \\
\text { Français moyen, étroit d'esprit, attaché à des } \\
\text { valeurs plus ou moins réactionnaires, voire } \\
\text { racistes. } \\
\text { Définition } 2(3 \%) \text { : } \\
\text { Type de Français moyen, réactionnaire et } \\
\text { raciste, inspiré d'un personnage de bandes } \\
\text { dessinées. } \\
\text { Définition } 3(42 \%) \text { : } \\
\text { Le beauf est un stéréotype de Français } \\
\text { moyen vulgaire, inculte et borné. } \\
\text { Définition } 4(39 \%) \text { : } \\
\text { Individu à l'esprit étroit, peu cultivé, souvent } \\
\text { vulgaire, sans gout et qui imite les autres. Le } \\
\text { beauf est peu cultivé et parfois fier de l'être : } \\
\text { la caricature d'un type de beauf le veut } \\
\text { empreint d'une certaine autosatisfaction. } \\
\text { Définition } 5(6,4 \%): \\
\text { Personne étroite d'esprit, conformiste à } \\
\text { l'extrême. }\end{array}$ & $\begin{array}{l}\text { (Femme, LLCE, } 20 \text { ans): } \\
\text { Un ringArd qui porte des chaussettes avec des } \\
\text { claquettes (ou des tongs), un short à fleurs } \\
\text { (accessoirement). Voir le personnage de Patrick } \\
\text { Chirac dAns Camping. } \\
\text { (Femme, LEA, } 19 \text { ans) : } \\
\text { il y a beaucoup l'idée de "has been" dans } \\
\text { beauf, c'est aussi synonyme de campagnard } \\
{[\ldots . .] \text {. }} \\
\text { (Femme, M1 linguistique, } 21 \text { ans): } \\
\text { mAl éduqués, ils becottent leurs meufs comme } \\
\text { une sucette dans la rue. }\end{array}$ \\
\hline Bolos(s) & $\begin{array}{l}\text { Définition } 1(2,2 \%) \text { : } \\
\text { Client du dealer de drogue. } \\
\text { Définition } 2(8,6 \%) \text { : } \\
\text { Personne d'apparence faible et sans défense } \\
\text { que l'on peut facilement gruger ou voler, } \\
\text { dupe. } \\
\text { Définition } 3(0 \%) \text { : } \\
\text { Personne peu sérieuse, fumiste. } \\
\text { Définition } 4(30 \%): \text { Injure adressée à un } \\
\text { individu que l'on dénigre en raison de son } \\
\text { statut social, de son manque de virilité, de } \\
\text { son manque d'amis, etc. } \\
\text { Définition } 5(7,5 \%): \\
\text { Un synonyme de victime, proie facile. } \\
\text { Définition } 6(6,4 \%): \\
\text { Quelqu'un de gentil, bien élevé voire cultivé } \\
\text { méprisé par ses pairs. } \\
\text { Définition } 7(26 \%): \\
\text { Nul, ringard ou encore démodé. } \\
\text { Définition } 8(18,3 \%): \text { Personne naïve ou peu } \\
\text { courageuse, au comportement ridicule, voire } \\
\text { stupide ; bouffon. }\end{array}$ & $\begin{array}{l}\text { (Femme, LLCE, } 18 \text { ans): } \\
\text { Les gens peuvent dire "bolos" juste parce } \\
\text { qu'ils n'aiment pas le physique de la personne } \\
\text { ou seulement pour mépriser. }\end{array}$ \\
\hline
\end{tabular}




\begin{tabular}{|c|c|c|}
\hline Crevard & $\begin{array}{l}\text { Définition } 1(3,6 \%) \text { : } \\
\text { Démuni, sans ressource. } \\
\text { Définition } 2(2,8 \%) \text { : } \\
\text { Affamé, goinfre. } \\
\text { Définition } 3(23 \%) \text { : } \\
\text { Avare, radin. } \\
\text { Définition } 4(0 \%) \text { : } \\
\text { Qui est d'aspect maladif, famélique. } \\
\text { Définition } 5(20,6 \%) \text { : } \\
\text { Au sens argotique, quelqu'un d'animé par de } \\
\text { viles intentions, qui agit lâchement. } \\
\text { Définition } 6(23 \%) \text { : } \\
\text { Avare; radin; égoïste; personne capable du } \\
\text { pire pour servir ses intérêts et qu'on } \\
\text { aimerait voir mourir. } \\
\text { Définition } 7 \text { (27\%): } \\
\text { Égoïste qui pense uniquement à ses intérêts } \\
\text { personnels et n'aide personne (surtout en ce } \\
\text { qui concerne l'aspect financier). }\end{array}$ & $\begin{array}{l}\text { (Femme, LLCE, } 20 \text { ans }) \text { : } \\
\text { Quand quelqu'un veut pas partager son grec/ } \\
\text { kebab; } \\
\text { (Homme, LLCE, } 18 \text { ans) }: \\
\text { Personne avare qui ne pense qu'à soi en } \\
\text { premier temps; } \\
\text { (Femme, LEA, } 19 \text { ans): } \\
\text { Par exemple, quand on sort entre amis et } \\
\text { qu'une personne n'a pas assez d'argent et } \\
\text { qu'un ami veut pas donner un peu d'argent, on } \\
\text { peut dire que c'est un crevard (pas méchant): } \\
\text { Tu ne veut pas m'avancer } 5 \text { euros, t'es un } \\
\text { crevard! } \\
\text { (Homme, LEA, } 19 \text { ans): } \\
\text { Ce n'est pas quelqu'un de serviable auquel on } \\
\text { peut rien demander pour quelques aides: Il ne } \\
\text { partage jamais, ce crevard! } \\
\text { (Femme, LLCE, } 20 \text { ans): } \\
\text { C'est aussi une insulte, genre ça peut être } \\
\text { synonyme de connard aussi. }\end{array}$ \\
\hline Kéké & $\begin{array}{l}\text { Définition } 1(34,2 \%) \text { : } \\
\text { Personne présomptueuse et vantarde } \\
\text { considérée comme ridicule par l'assistance. } \\
\text { Définition } 2(21 \%) \text { : } \\
\text { Régional (Est, Sud-Est). Crâneur ; idiot. } \\
\text { Définition } 3(44,8 \%) \text { : } \\
\text { Individu qui cherche à impressionner par } \\
\text { son comportement et s'avérant ridicule et } \\
\text { lourd. }\end{array}$ & $\begin{array}{l}\text { Kéké des plages (expression proposée par } \\
\text { plusieurs interrogés): } \\
\text { Kéké des plages: gars skinny qui croit qu'il va } \\
\text { devenir hulk en } 2 \text { jours. (EF613). }\end{array}$ \\
\hline Kikoo & $\begin{array}{l}\text { Définition } 1(76 \%) \text { : } \\
\text { Adolescent abusant des abréviations et sans } \\
\text { réflexion quant au contenu ou à la portée de } \\
\text { ses SMS. } \\
\text { Définition } 2(0 \%) \text { : } \\
\text { Personne stupide qui se fait toujours avoir. } \\
\text { Définition } 3(24 \%) \text { : Gamin. }\end{array}$ & $\begin{array}{l}\text { (Femme, LLCE, 19 ans) }: \\
\text { Très péjoratif sur le web. Personne modifiant } \\
\text { son écriture et l'ortographe des mots (ma } \\
\text { sistahhh). } \\
\text { (Femme, LEA, } 18 \text { ans) }: \\
\text { Quelqu'un qui fait gamin par rapport à son âge } \\
\text { et par rapport à ce qu'il fait. } \\
\text { (Femme, ScPO, 19 ans) : } \\
\text { Personne qui ne réfléchit pas à ses propos qui a } \\
\text { des réactions disproportionnées sur internet. } \\
\text { (Femme, M1 linguistique, 22 ans): Jeune } \\
\text { personne se croyant mature dans une situation } \\
\text { sociale. }\end{array}$ \\
\hline
\end{tabular}


Il apparait évident que tous les items ont connu une extension du sens premier, fait qui est rarement mentionné dans les ouvrages dictionnairiques que nous avons consultés. Compte tenu des précisions fournies par nos interrogés sur le potentiel connotatif des items étudiés et des exemples illustratifs de leur fonctionnement contextuel, nous avons pu dégager plusieurs tendances.

\subsection{Fonctionnement socio/ontotypique des termes étudiés}

Notons globalement que, selon nos participants, tous les lexèmes, mis à part la valeur dominante péjorative, peuvent acquérir d'autres connotations (humoristique, ironique, affective) dans différents contextes énonciatifs. Nous nous intéresserons maintenant aux trois types d'emplois distingués.

\subsubsection{Référence à la classe sociale (emploi idéologique/sociotypique)}

Dans ce type d'emploi, les items fonctionnent dans leur sens plein, sont souvent renforcés par d'autres éléments du contexte et, dans l'ensemble, visent à exprimer le contenu méprisant. La stratégie discursive de mépris se déploie ici par la mise en place du processus simultané de dévalorisation de l'autre et de valorisation de soi (Bres, 1993). Dans les exemples fournis, le locuteur a recours à une sorte de sociotypification, il construit une image prototypique d'un représentant d'une classe bien déterminée. Ce représentant est évalué selon une de ses caractéristiques qui permet de le catégoriser comme beauf, cassos, bolos, crevard, kéké ou kikoo.

Dans les exemples (2) à (5), un des traits propres au beauf sert de motif à partir duquel le locuteur réalise un acte de mépris. Il s'agit du style vestimentaire en (2), de la façon de considérer les choses en(3), des caractéristiques physiques en (4) et du comportement inapproprié en (5).

(2)

Il porte une chemise de beauf avec des claquettes et des chaussette.

(3)

T'as entendu ce qu'il a dit ? C'est vraiment un beauf !

(4)

Thibault Roquiny mdrr j'en peux plus de sa gueule de beauf pfffff [ERS28].

(5)

C'est pas un daron, c'est un beauf devant son PC [EF29].

Dans ce genre de contexte, nous voyons se construire un sociotype ayant des caractéristiques socioculturelles ou idéologiques communément admises. En ce qui concerne l'aspect lexiculturel, quelques références nous ont été également proposées par les enquêtés, dont le personnage de Patrick Chirac interprété par Franck Dubosc dans Camping, une série de films français diffusée en 2006. Si l'on parcourt rapidement les critiques de presse disponibles en ligne, nous trouvons quelques suggestions intéressantes. Attali, dans Le Monde, affirme qu' « un choc des cultures et un échantillon d'humanité sont les leviers de ce scénario aussi éphémère qu'un château de sable où le beauf est roi ". La rédaction de Libération estime que ce film donne une image de "la beauferie française ». Dans la même veine, Télécinéobs considère qu'il s'agit d'« un portrait de la beaufitude entre cruauté et tendresse » et Chronicart remarque que ce film ré-oxygène "le genre moribond de la comédie sociologique ». Le mode de vie de certaines classes de la société française fait donc l'objet d'une interprétation satirique et d'une 
sorte de sociotypification évoquée auparavant. Nous sommes en présence de la mobilisation d'un aspect socioculturel, car si ce beauf devient un personnage-cible des caméras des cinéastes, c'est parce que le cinéma, comme toute autre production culturelle, est un miroir de la société. Tous les attributs stéréotypés propres aux beaufs sont évidemment repris dans la création du personnage principal pour que les spectateurs reconnaissent bien le type. Si l'on effectue une brève recherche sur les forums (notamment sur Blabla 18-25 ans) et sur Twitter qui évoquent le sujet, nous retrouverons également quelques traits comportementaux distinctifs, le beauf étant aussi « celui qui rigole très fort en se tapant les cuisses, qui fait le tuning de sa voiture, qui boit beaucoup d'alcool, qui préfère un humour vulgaire (qui "note et commente ses pets et ses rots"), qui est macho ". Par référence à la classe sociale le locuteur vise à se distinguer de l'image dévalorisante qu'il évoque en produisant un ethos discursif méprisant.

Il en va de même pour les autres lexèmes. Dans l'exemple (6), boloss est employé en son sens plein. Il est renforcé par plusieurs autres éléments : les axiologiques négatifs et les adjectifs disqualifiants comme gros nerds et un selfie dégueulasse qui contribuent à la réalisation d'un acte de mépris.

(6)

[...] franchement une fois j'ai regardé les profils des mecs rapidement, y'a tellement de boloss on se rend pas compte genre des gros nerds avec un selfie dégueulasse et une description de 20 lignes (EF211).

En (7) et (8), la portée péjorative des exemples fournis vise à mettre en relief le caractère avare de la personne délocutive.

(7)

Tu ne veut pas m'avancer 5 euros, t'es un crevard !

(8)

Il ne partage jamais, ce crevard !

Nous avons également relevé des exemples semblables dans le corpus; en voici un :

(9)

L1 : Moi je suis fumeur, souvent quand on me demande une clope j'en donne une, c'est 30 cents donc ça a très peu de valeur. J'ai cru que tout le monde faisait ça mais je me suis rendu compte que non en fait. Dimanche dernier j'étais en galère de clope donc j'ai quémandé devant un ciné, j'ai essuyé que des refus, wallah j'étais choqué !!! C'est quoi le problème de ces gens qui donne pas ??? Ils sont jamais en galère ou quoi ? la notion de partage, ils connaissent?

L2: ils te sauvent la vie c'est des heros wesh

L3 : Ils pensent a ta santé mdr

L1: ya pas c'est des héros c'est des crevards mon gars (EF116)

Dans l'exemple (9), le mécanisme discursif du mépris s'effectue en deux étapes: la première, à double visée, sert de motif du jugement posé par la suite et en même temps de moyen de valorisation de soi. En disant «quand on me demande une clope j'en donne une ", le locuteur projette un ethos de générosité et de bienveillance et en profite pour poser en amont un motif pour lequel il traitera ultérieurement la personne délocutive de crevard. La deuxième partie vise à démontrer que le fait que l'autre est différent du même et sachant que le même n'est pas crevard, l'autrui est estimé l'être. Le locuteur construit donc une image valorisante de lui qui sert de critère de la dévalorisation de l'autre. Le mécanisme simultané de valorisation-dévalorisation étant mis en place, nous pouvons considérer ce discours comme relevant du mépris. Citons encore un exemple où le jugement dévaluatif porte sur le caractère égoïste de la 
personne délocutive, et non pas avare cette fois-ci, et se construit explicitement par opposition à l'autre :

(10)

Je vois bien le crevard qui se pointe tôt le matin pour faire son petit tour de charognard et rafler tout ce qu'il trouve sans penser aux autres (EF115).

En ce qui concerne l'item kéké, il est complexe et culturellement chargé. Rappelons que l'aspect idéologico-culturel est caractéristique des sociotypes et déterminant pour la réalisation de l'acte de mépris. Dans l'exemple (11), le lexème est employé en son sens plein et exprime en lui seul, sans aucun renforcement contextuel, le contenu méprisant.

(11)

L1: Tu payes combien?

Je suis sûr qu'il y a plein de kéké dans ta salle

L2 : Ahahahhhhh Arrête de critiquer ma salle ! $35 €$. Et toi ?

L1 : Jure ?! Je paye $20 €$, et ya pas bcp de mec qui se la pète, genre en marcel en train

de se regarder devant la glace ${ }^{\wedge \wedge}$ (ERS432)

Notons que la séquence kéké des plages, que nous voyons apparaitre plusieurs fois dans le corpus, semblerait fonctionner comme une expression toute faite à valeur emblématique. Une fois de plus, nous sommes en présence d'une sociotypification, procédé par lequel se construisent deux profils différents : d'un côté, celui de kéké et de l'autre, celui de go muscu, une paraphrase pour désigner des passionnés de musculation qui en font régulièrement, correctement et ne considèrent pas le sport comme une simple passion, mais un mode de vie. Voici les propos d'un des internautes :

(12)

Alors kéké des plages ... gars skinny qui croit qu'il va devenir hulk en 2 jours. Ta le kéké tout maigre et le kéké gros. Le kéké gros est une espèce plus ou moins commune dans les salles de musculation, c'est surtout celui qui va se croire badass avec son $30 \mathrm{~cm}$ de tour de gras. Il pousse des cris, il triche, il soulève "lourd" croyant que c'est une base. Tu as le kéké skinny moins commun mais bien existant qui se croit bg car il voit ses muscles à ras de ses os. Oui je sais, ils sont vraiment naïf (EF613).

À l'opposé se retrouvent donc les kékés qui, comme l'affirme un de nos participants, « passent leur temps à rouler des mécaniques devant les jolies filles ».

J'allais go muscu maintenant faire ma séance push mais je me suis rappelé des kékés des plages. J'attends 20h (EF615).

(14)

L1: Pourquoi les go muscu ont la rage contre le kéké des plages?

L2 : Car ils pratiquent que 1 ete dans le but $d$ attirer les femmes et croient que des pompres abdo va les rendre balaise (EF618).

(15)

Je vais t'expliquer pourquoi je vais à la salle, petit donneur de leçon derrière ton Pc [...] car c'est bénéfique pour moi , depuis que je fais de la muscu je fais attention à ce que je mange ( pas dans l'extrême non plus hein, je me prive pas au resto et au fast food avec des meufs ou des collegues, je ne suis pas dans le cliché du Tupperware loin de la), j'ai une plus grande détermination à accomplir mes objectifs de la vie quotidienne, je suis plus heureux car plus épanouie sociolament vu que j'ai confiance en moi [...] (EF604).

Dans les exemples (12) à (15), le mécanisme de mépris se construit autour du profil du locuteur. Si celui-ci s'identifie à un groupe de "go muscu ", l'instance discursive qu'il produit par son dire s'oppose idéologiquement parlant de celui de kéké. Comme 
l'affirment Reynolds, Turner et Haslam (2003), un individu qui s'identifie fortement à un groupe donné risque également de se conformer sans discussion à un comportement stéréotypé qui a été déjà prescrit par ce groupe de référence. Dès lors le mécanisme simultané de dévalorisation-valorisation se déclenche et l'item kéké fonctionne comme sociotype et revêt dans le contexte une forte valeur de mépris. Ces deux appellations fonctionneraient comme des badges catégorisant des représentants en fonction de leur appartenance à un groupe qui est ici revendiquée. Le terme de badge est emprunté à Conein (2017) qui affirme que " proclamer ou affirmer un nom de groupe en première personne revient à valoriser une identité, en faire un badge ou un emblème " (p. 149-150).

Il en va de même pour kikoo. Dans l'exemple (16), l'item fonctionne comme sociotype et sert à poser un jugement de valeur méprisant. Les séquences perdre ton temps, c'est vraiment un gamin, bloque le prouvent que le locuteur vise à se distinguer de la personne délocutive en laissant entendre "contrairement à lui, nous ne sommes pas gamins, nous n'écrivons pas comme des kikoo». En même temps, le nous qui est ici sousentendu témoigne d'une stratégie de connivence : par la dévalorisation de la catégorie des « kikoo ", le locuteur cherche à positionner son représentant (personne délocutive) comme autrui idéologique tout en instaurant une connivence interpersonnelle avec son interlocuteur (la reconnaissance de ses mêmes, appartenant à une autre catégorie supposée être « au-dessus »).

(16)

Regarde déjà comment il écrit. C'est un kikoo. Non mais bloque le hein, c'est vraiment un gamin. Tu vas pas perdre ton temps avec ca quand même (ERS235).

\subsubsection{Emplois axiologiquement ambivalents : glissements sémantiques et valeurs}

41 Plusieurs emplois différents seront regroupés ici. Dans tous les cas, il s'agit d'emplois ontotypiques, soit d'exemples où le sens des termes étudiés s'étend. De ce fait, nous les observerons fonctionner dans des contextes axiologiquement polaires en tant qu'ontotypes. Rappelons qu'à la différence des sociotypes, les ontotypes ne renvoient pas simplement à une classe, ils sont nécessairement évaluatifs : fortement liés à la situation d'énonciation, ils sont employés à visée ironique, humoristique, autodévalorisante ou méprisante selon les paramètres de la situation d'énonciation; dans ce type d'emploi, nous verrons se développer plusieurs stratégies discursives relevant du mépris, du blocage de mépris, du mépris délocutif.

42 Nous avons vu jusqu'ici que pour exprimer son mépris, le locuteur vise la valorisation du même et la dévalorisation de l'autre. Ici, nous nous consacrerons à l'analyse des extraits de notre corpus où l'image de soi est intentionnellement détériorée à visée humoristique ou ironique. Ce type de fonctionnement discursif prouve que les items étudiés connaissent bien des emplois à portée auto-dévaluative. C'est notamment le cas de cassos dans les exemples ci-dessous :

(17)

L1 : [...] du coup je $\mathrm{m}(\mathrm{e})$ suis ramenée habillée en cassos [...]

L2 : (Rire) comme d'hab(itude) (0046).

(18)

De toute façon je suis une cassos des relations sentimentales mdrr (EF321).

En (17), le locuteur n'appartenant pas à la catégorie des cas sociaux au plein sens du terme et ne s'attribuant qu'une seule caractéristique (externe) de la catégorie, 
l'humour est fondé sur le décalage entre le dit et la réalité pour provoquer une réaction souhaitée, le rire. Le syntagme habillée en cassos aurait un rapport avec la situation financière difficile des gens appartenant à la catégorie sociale en question et signifie implicitement "habillée modestement ». L'ethos intentionnellement dégradé qui est rendu par le discours du locuteur ne dévalorise pas une personne en présence dans cette situation d'énonciation précise (la conversation se déroule entre deux étudiantes issues de la classe moyenne). Le message est donc décodé correctement et peut être considéré comme réussi, car il a atteint un effet prévu, le rire. Néanmoins, le même message aurait provoqué une réaction différente si un paramètre avait été changé, par exemple s'il avait été émis lors d'un échange avec un interlocuteur qui appartient à cette catégorie sociale. Dans ce cas-là, le message habillée en cassos aurait été incorporé différemment : il aurait probablement été perçu comme méprisant, car l'interlocuteur se serait senti dominé. Pour revenir à l'exemple initial, notons qu'il pourrait s'agir d'une forme de mépris "délocutif ", de mépris de classe qui émergerait par rapport à des représentants de la catégorie sociale en question, mais elle ne serait pas intentionnelle dans ce type d'emploi à portée humoristique.

Dans l'exemple (19), le jugement évaluatif est porté sur soi :

(19)

ouais ouais je fais trop le beauf là mais j'assume hein ma passion pour le sport auto, l'amour du cinéma de genre (série $Z$ ), et d'autres trucs bannis par l'intelligentsia (EF174).

Le locuteur présuppose que ses passions, caractéristiques des gouts et des pratiques des "beaufs", seraient probablement mal vues et adopte une stratégie d'anticipation pour présenter un ethos conscient et confiant. Le rabaissement de soi atteint un effet inverse : la valorisation de soi sous forme de pseudo-auto-disqualification. Le locuteur affiche la posture dominante d'un homme sûr de lui qui ne voit rien de honteux dans ses habitudes que la conscience façonnée d'autrui pourrait catégoriser comme relevant de la «beauferie ». C'est une sorte de contre-attaque (définie comme prolepse par Perelman \& Olbrechts-Tyteca, $1983^{10}$ ) qui bloque un éventuel acte de mépris, car tant que la cible ne le perçoit comme tel, il n'aurait pas cet effet.

En ce qui concerne l'item boloss, il a également connu une extension axiologique. Les exemples prouvent que son emploi tend vers la catégorie d'ontotype, car il apparait dans des contextes axiologiquement différents : péjoratifs, humoristiques ou ironiques. Évoquons le commentaire d'un des interrogés pour appuyer notre observation : «Très péjoratif. Insulte adressée à une personne sans forcément connaitre la personne. Peut être utilisé de façon humoristique entre amis, à la suite d'une mauvaise blague, d'une idée stupide dans des phrases du genre: "Victime boloss!/ Tu t'es trompé de bus? Ahah quel boloss !" " Nous en déduisons que le contenu sémantique dominant peut être effacé dans certains environnements scénographiques au profit d'une valeur ajoutée, comme nous l'avons vu pour cassos. Selon un de nos enquêtés, "les gens peuvent dire "bolos" juste parce qu'ils n'aiment pas le physique de la personne ou seulement pour mépriser ». Un commentaire signale également qu'il s'agit d'un « mot utilisé par des personnes bas de plafond». Nous insistons donc sur le fait que l'orientation axiologique des énoncés dépend des valeurs morales, comportementales et idéologiques du sujet parlant, de la situation d'énonciation englobante et de la relation établie entre les locuteurs et la personne délocutive. Autrement dit, tout dépend de l'échelle de valeurs du sujet parlant. Elle est étroitement liée à la notion de norme et régie par la confrontation des objets d'évaluation avec les critères normatifs. 
Notons au passage que le dernier commentaire relève du mépris, car l'énonciateur produit un ethos s'élevant au-dessus des autres (des personnes bas de plafond).

En résumé, ce type d'emploi démontre une souplesse axiologique propre aux lexèmes étudiés, qui apparaissent dans des contextes polaires. Ajoutons, suivant Lagorgette et Larrivée (2004), que tout axiologique négatif peut être relativisé par le contexte et employé à des fins affectives. Nous avons relevé de nombreuses attestations de l'emploi où la charge négative du message est neutralisée par divers adoucisseurs. Les exemples de ce retournement en contre-discours affectif seront présentés à la section suivante.

\subsubsection{Emplois ontotypiques de solidarité}

Nous avons regroupé ici des emplois hypocoristiques typiques des contextes de connivence. Actualisés dans des discours à portée affective, les items étudiés perdent leur sens dominant, entrainant un changement radical de scénographie énonciative : nous assistons à des échanges entre amis qui s'entendent bien et qui peuvent se permettre de recourir à l'implicite. Notre réflexion rejoint celle de Meillet (1905), qui postule que

dans certaines conditions n'importe quel élément complémentaire peut devenir plus important, quelquefois même à un point tel qu'il devient dominant tandis que, en conséquence, la dominante originaire recule à l'arrière-plan et ne représente plus qu'un des éléments complémentaires (p. 47-48).

Il ajoute qu'on peut observer assez fréquemment une perte absolue de la dominante dans la signification des mots exprimant la tendresse et des injures, puisque « la force des sentiments fait remplacer un mot par un autre mot d'un sens différent, n'ayant parfois rien de commun avec le sens en question ». Ainsi, le locuteur a l'intention " soit de voiler la dominante, soit de mettre en lumière l'un des éléments complémentaires et surtout employer un mot plus expressif, inaccoutumé et inattendu ». Nous observerons ici des emplois où les items perdent leur dominante (leur contenu méprisant) et fonctionnent comme des affects ${ }^{11}$ :

(27)

mdrrrrr je m'etouffe vilain crevard va ;) (EF515)

(28)

Je meurs, mon ami c'est un cassos quoi

(29)

Salut les boloss ça va ou quoi (OR12)

(30)

Heyy, Kikoo ça va ? C bosse dur askip mdr (ER345).

\section{Conclusion}

Cette analyse montre que les lexèmes étudiés ont des traits sociotypiques et ontotypiques qui les rendent complexes et chargés d'un point de vue sémantique, conceptuel et pragmatique. Ce vocabulaire à substrat idéologique et socioculturel peut être actualisé dans des contextes à portée condescendante afin de traduire le mépris que le sujet parlant éprouve envers autrui en construisant par son discours un ethos dominant. Dans ces cas de figure, où il est question d'un acte de mépris, le recours à ces lexèmes sert de moyen de se valoriser et de rabaisser autrui sur le plan social (le cas de cassos, beauf, crevard et bolos) ou idéologique (le cas de kéké et kikoo). Ils sont chargés 
culturellement: nos interrogés ont fourni plusieurs informations lexiculturelles qui précisent et déterminent leur apparition dans des contextes à visée méprisante.

51 Ainsi, nous avons distingué des conditions nécessaires pour la réalisation d'un acte de mépris: d'abord, les lexèmes doivent fonctionner comme sociotypiques, car l'actualisation de la dimension idéologique ou socioculturelle est cruciale pour tout acte de mépris ; ensuite, la stratégie discursive de mépris se caractérise par une simultanéité du fonctionnement de deux mécanismes, celui de valorisation de soi et de détérioration de l'image de l'autre ; enfin, la troisième condition est étroitement liée à la deuxième et exige que ce double mouvement d'axiologisation soit basé sur un motif explicite pour l'objet-cible. Étant donné qu'il s'agit d'un jugement que le sujet parlant pose sur autrui, il doit être fait à partir d'une norme communément admise par tous les concernés. Soulignons que l'acte de mépris requiert des stratégies discursives complexes afin d'être perçues comme tel et d'atteindre un effet pragmatique souhaité. Néanmoins, il existe également des environnements contextuels où le sens dominant des lexèmes est relativisé. Ils se comportent alors comme ontotypiques ayant une valeur d'insulte ou d'affectif et perdent leur valeur de mépris.

\section{BIBLIOGRAPHIE}

ALSAFAR, Ali. (2014). Ethos discursif et construction des rapports intersubjectifs dans les professions de foi des élections présidentielles de 2007 et de 2012 (Thèse de doctorat, Université Paul Valéry -

Montpellier 3). Disponible en ligne sur <https://tel.archives-ouvertes.fr/tel-01146590/document> (consulté le 20 avril 2019).

Amossy, Ruth. (2010). La Présentation de soi : ethos et identité verbale. Paris : Presses universitaires de France.

AMOssy, Ruth. (2014). Apologie de la polémique. Paris : PUF.

BRES, Jacques. (1993). Récit oral et production d’identité sociale. Montpellier : Université de Montpellier, Praxiling.

Colin, Jean-Paul, MÉVEL Jean-Pierre \& LECLÈRE Christian. (2006). Argot \& français populaire : grand dictionnaire. Paris : Larousse.

CONEIN, Bernard. (2017). Deux manières d'énoncer une identité. Dans F. Gadet et coll. (dir.), Les parlers jeunes dans l'île-de-France multiculturelle (p. 143-159). Paris : Ophrys.

DICTIONNAIRE DE FRANÇAIS LAROUSSE. (2019). Dictionnaire français - Dictionnaires Larousse français monolingue et bilingues en ligne. Disponible en ligne sur <www.larousse.fr/dictionnaires/francais> (consulté le 20 avril 2019).

DictionnAire de LA ZONE. TOUT L'ARgot des BAnlieues. (2000). Dictionnaire. Le dictionnaire de la Zone. Disponible en ligne sur <www.dictionnairedelazone.fr/dictionary/definition> (consulté le 20 avril 2019). 


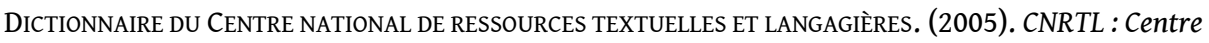
national de ressources textuelles et lexicales - Dictionnaires modernes. Disponible en ligne sur $<$ www.cnrtl.fr/dictionnaires/modernes/> (consulté le 20 avril 2019).

ERNOTTE, Philippe \& ROSIER, Laurence. (2004). L'ontotype : une sous-catégorie pertinente pour classer les insultes? Langue française, 144, 35-48.

HARE, Robert. (1999). Without Conscience: The Disturbing World of the Psychopaths Among Us. New York : Guilford Press.

JUIGNET, Patrick. (2016). Quelle ontologie proposer aujourd'hui ? Philosophie, science et société. Disponible en ligne sur <https://philosciences.com/philosophie-generale/ontologie-reel-realite/ 195-existence-reel-realite> (consulté le 2 septembre 2019).

LABOV, William. (1972). Rules for Ritual Insults. Dans D. Sudnow (dir.), Studies in Social Interaction (p. 120-170). New York : Free Press.

LAGORGETTE, Dominique \& LARRIVÉE, Pierre. (2004). Interprétation des insultes et relations de solidarité. Langue française, 144, 83-103.

LAGORGETTE, Dominique. (2007). Termes d'adresse et insultes : discours sur l'autre ou sur moi ? Dans W. Ayres-Benett \& M. C. Jones (dir.), The French Language and Questions of Identity (p. 116-128). Londres : MHRA Legenda.

LePoutRe, David. (2001). Cœur de banlieue. Codes, rites et langages. Paris : Odile Jacob.

MAIngueneau, Dominique. (2009). Les termes clés de l'analyse du discours. Paris : Seuil.

MeILlET, Antoine. (1905). Comment les mots changent de sens. Année sociologique 1905-1906, 16-20.

MicheLI, Raphaël. (2014). Les émotions dans les discours : modèle d'analyse, perspectives empiriques.

Paris : De Boeck.

MoÏsE, Claudine. (2004). Postures sociales, violence verbale et difficile médiation. Dans

R. Delamotte-Legrand (dir.), Les médiations langagières : actes du colloque de Rouen (p. 335-349).

Rouen : Presses de l'université de Rouen.

Mougin, Sylvie. (2006). La médisance. Reims : Presses universitaires de Reims.

Perelman, Chaïm \& Olbrechts-TyteCA, Lucie. (1983). Traité de l'argumentation. La nouvelle rhétorique. Bruxelles : Presses de l'Université de Bruxelles.

PERRY, Ralph Barton. (1926). General Theory of Value. Cambridge : Harvard University Press.

REYNOLDS, Katherine, TURNER, John \& HASLAM, Alexander. (2003). Social Identity and SelfCategorization Theories' Contribution to Understanding Identification, Salience and Diversity in Teams and Organizations. Research on Managing Groups and Teams, 5, 279-304.

RosIER, Laurence. (2006). Petit traité de l'injure. Loverval : Labor.

SECA, Jean-Marie. (2012). Idéologie, représentations sociales et urbanité. Les cahiers psychologie politique. Paris : Armand Colin. Disponible en ligne sur <http://lodel.irevues.inist.fr/ cahierspsychologiepolitique/index.php?id=2002> (consulté le 2 septembre 2019).

VINCENT, Diana \& HEISLER, Troy. (1999). L'anticipation d'objections : prolepse, concession et réfutation dans la langue spontanée. Revue québécoise de linguistique, 27(1), 15-31. 


\section{NOTES}

1. Collectées par observation participante.

2. Les données « écologiques » ont été soumises à la transcription. Pour les données écrites, nous avons respecté l'écriture attestée avec la ponctuation, les erreurs d'orthographe et les fautes de frappe.

3. Dictionnaire Larousse en ligne: <www.larousse.fr/dictionnaires/francais/mépris/50556? q=mépris\#50445>. C'est nous qui soulignons à l'intérieur de la citation

4. D'autant plus qu'un des mots-clés de la définition citée est moralement qui mérite d'être nuancé, car ce qui est moralement admis pour l'un n'est pas systématiquement accepté par l'autre.

5. Pour reprendre les termes d'Amossy (2010).

6. Voir à ce sujet Moïse (2004), notamment pour le cas des vannes.

7. Dans des contextes du type : «l'examen n'a pas été trop dur, fais pas ton babtou ».

8. Nous avons eu recours au dictionnaire de Colin, Mével et Leclère (2006), au Dictionnaire de la zone. Tout l'argot des banlieues (2000) et au Dictionnaire du Centre national de ressources textuelles et lexicales (2005).

9. Ici et plus loin, les commentaires explicatifs proposés par les enquêtés afin de préciser ou nuancer le potentiel distributionnel et connotatif des termes étudiés ne sont pas numérotés, contrairement aux exemples d'emploi.

10. Voir également Vincent et Heisler (1999) à ce sujet.

11. Voir notamment à ce sujet, Labov (1978), Lepoutre (2001) et Moïse (2004), pour le cas des vannes et des insultes rituelles.

\section{RÉSUMÉS}

Les émotions dans le discours occupent depuis quelques années une place de choix dans de nombreuses études. Pourtant le mépris n'a jamais fait l'objet d'une analyse pluridimensionnelle détaillée. L'acte de mépris est un phénomène discursif complexe qui renvoie à la sphère des valeurs et qui requiert des mécanismes particuliers afin de pouvoir aboutir et atteindre sa cible. Notre texte portera sur l'emploi de termes potentiellement interprétables comme méprisants et dépositaires de traces de stéréotypes socioculturels (cassos, beauf, bolos, crevard, kéké et kikoo) dans de différents environnements axiologiquement polarisés. Nous essaierons de saisir comment distinguer l'acte de mépriser d'autres actes du discours en relevant ses caractéristiques pragmatiques aussi bien que les effets qu'il est susceptible de provoquer chez soi et chez l'autre.

This article presents an analysis of several terms often used in the youth student environment, which, outside the context, are often regarded as evaluative, expressing the contemptuous attitude of the speaker to the subject of speech and being a kind of social-cultural stereotypes (cassos, beauf, bolos, crevard, kéké and kikoo). Based on data from two corpora of young students' speech (oral and written) and the sociolinguistic questionnaire, the article attempts to identify the main discursive mechanisms of expressing contempt and the specific contexts where the same lexemes express axiologically polar semantic nuances. 
INDEX

Mots-clés : stratégies discursives, mépris, évaluation ambivalente, emploi ontotypique

Keywords : discursive strategies, contempt, ambivalent evaluation, ontotype use

\section{AUTEUR}

POLINA UKHOVA

Université de Poitiers 